\title{
Solar glint from uncoated starshade optical edges
}

\author{
Stuart Shaklan $\odot *$ Evan Hilgemann $\odot$, Dylan McKeithen, \\ Stefan R. Martin $\odot$, John Steeves $\odot$, David Webb, and Douglas Lisman \\ Jet Propulsion Laboratory, California Institute of Technology, Pasadena, California, \\ United States
}

\begin{abstract}
The perimeter of a sunflower-like starshade has hundreds of meters of sharp edges that are directly exposed to sunlight. The sunlight diffracts and reflects from the edge resulting in a dual-lobed glint pattern that can be brighter than an exoplanet. We present estimates of the glint brightness distribution for the Starshade Rendezvous Mission and the HabEx Starshade Mission concepts based on measurements of flight-like, environmentally tested, uncoated metallic edges using custom-built scatterometers. A companion paper addresses the performance for edges coated with a thin anti-reflection coating. (C) The Authors. Published by SPIE under a Creative Commons Attribution 4.0 Unported License. Distribution or reproduction of this work in whole or in part requires full attribution of the original publication, including its DOI. [DOI: 10.1117/1.JATIS.7.2.021204]
\end{abstract}

Keywords: starshade; glint; exoplanet imaging; scatterometry.

Paper 20116SS received Aug. 1, 2020; accepted for publication Nov. 6, 2020; published online Jan. 8, 2021.

\section{Introduction}

When a space telescope observes a star, the proximal scattered light caused by diffraction, aberrations, surface irregularities, and contaminants is typically $\sim 10^{-3}$ to $10^{-5}$ times as bright as the star itself. This "veiling glare" prevents the observation of the faint reflected-light from exoplanets that would typically be $\sim 10^{-9}$ to $10^{-10}$ times fainter than the star.

A starshade positioned between the telescope and the star is designed to form a deep shadow around the telescope. This enables the observation of exoplanets with a high quality but otherwise unspecialized telescope. The starshade's flower-like shape controls starlight diffraction without blocking the view to the planet. ${ }^{1}$ The geometry calls for a starshade that is tens of meters in diameter while positioned tens of thousands of kilometers in front of the telescope. Sunlight must not strike the telescope-facing side of the starshade as this would reintroduce glare that is orders of magnitude brighter than the exoplanet. With the Sun positioned in the target star-facing hemisphere of the starshade (Fig. 1), taking care not to allow sunlight into the telescope aperture, the sunlight does not illuminate the telescope-facing surface but it does glint, i.e., reflect and diffract, off the starshade's sharp edges.

Steeves et al. ${ }^{2}$ measured the scatter for a variety of edges, including sharp metallic razor-like edges, and sharp edges coated or treated with dark materials, such as Acktar Black, ${ }^{3}$ as well as edges machined from pyrolytic graphite, an inherently dark material. They found that the uncoated, sharp metallic edges with a terminal radius of $\sim 150 \mathrm{~nm}$ performed better than the black-coated edges whose radii were $\sim 10$ to $30 \mu \mathrm{m}$. In the trade-off between surface area and reflectivity, the razor-like edges had superior scattered light performance. We have shown that sharp metallic edges approach the Sommerfeld diffraction limit ${ }^{4}$ and verified this with several models and laboratory measurements. ${ }^{5}$

In this paper, we focus on the optical testing of glint from the starshade edges, the manufacture and environmental testing of the edges, and the predicted on-sky appearance of the glint. We consider two mission concepts: the Starshade Rendezvous Mission (SRM) has a 26-mdiameter starshade in formation with the Roman Space Telescope, ${ }^{6}$ and the HabEx Mission ${ }^{7}$ utilizes a 52-m-diameter starshade. As shown in Table 1, SRM has two bandpasses, referred to as "blue" and "green," that require the starshade to be 37.2 and $25.7 \mathrm{Mm}$ in front of the

*Address all correspondence to Stuart Shaklan, stuart.b.shaklan@jpl.nasa.gov 


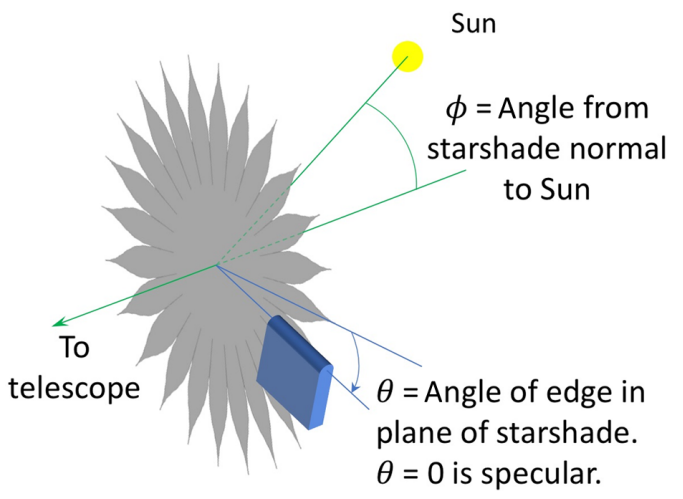

Fig. 1 Orientation of the Sun, starshade, telescope, and a segment of optical edge that is glinting. The edge orientation angle is $\theta$, and the Sun angle is restricted to $40^{\circ}<\phi<83^{\circ}$.

Table 1 Starshade missions.

\begin{tabular}{lcccc}
\hline \hline Mission & Bandpass $(\mathrm{nm})$ & Starshade diameter $(\mathrm{m})$ & Distance $(\mathrm{Mm})$ & Star-Sun angle $\left(^{\circ}\right)$ \\
\hline SRM (blue) & 425 to 552 & 26 & 37.2 & 54 to 83 \\
SRM (green) & 615 to 800 & 26 & 25.7 & 54 to 83 \\
HabEx & 300 to 1000 & 52 & 76 & 40 to 83 \\
\hline \hline
\end{tabular}

telescope, respectively. The HabEx mission is designed to have a larger instantaneous bandpass with a single-distance starshade at $76 \mathrm{Mm}$. The range of Sun angles is limited the low range by the telescope baffles and at the high end by the starshade's telescope-facing structure and alignment tolerances.

Section 2 describes two custom-built scatterometers we use to measure the glint on 50-mmlong edge coupons as well as on flight-like 50-cm-long shaped edge segments. The measurements produce a 2-D scatter distribution function (SDF) over the range of Sun angles applicable to the starshade missions.

Next, in Sec. 3, we show how the SDF is employed by our starshade imaging simulation package called SISTER ${ }^{8}$ to predict the appearance of the glint lobes. We also describe how the code was validated against theoretical calculations for a pure diffracting edge.

Section 4 describes the flight optical edge design and prototypes that consist of a photochemically etched amorphous metal (AM) working edge bonded to a carbon fiber reinforced polymer (CFRP) substrate. The etching process creates a sharp terminal edge that both limits solar glint and defines the precise in-plane shape. We have built prototypes and exposed them to representative flight-like thermal and deployment cycles described in Sec. 5.

The results of optical scatter testing and the experimental error budget are reported in Sec. 6 followed by the predicted visual magnitude of the solar glint lobes for SRM and HabEx in Sec. 7. We show that for the uncoated edges, the average brightness at the starshade's inner working angle (IWA), defined as the angle from the center to the tips of the starshade as seen from the telescope, is $\sim V=25$ for SRM and $\sim V=27$ for HabEx. In a companion paper, we report on new results using thin hybrid dielectric-interferometric coatings that greatly improve performance. ${ }^{9}$

This work is largely based on the experimental work performed for Milestone 3 of the Starshade Technology Program. ${ }^{10}$ The final report ${ }^{11}$ has been accepted by the Exoplanet Exploration Program Technical Advisory Committee. ${ }^{12}$

\section{Measuring Glint in the Laboratory}

We have built two instruments to measure the scatter function from optical edges. The first, the multi-angle scatterometer (MAS), ${ }^{13}$ was built several years ago and measures the full 2-D SDF 
of 3-mm-long sections of 50-mm-long coupons. The second, the single-angle scatterometer (SAS), ${ }^{14}$ was built in 2018 to 2019 to measure the integrated scatter over a fixed full-cone angle of $30^{\circ}$ along the $500-\mathrm{mm}$ length of curved optical edge assemblies.

\subsection{Multi-Angle Scatterometer}

The MAS was designed to measure scatter performance of coupon-scale components (roughly $25 \mathrm{~mm} \times 50 \mathrm{~mm}$, Fig. 2) over the full range of possible Sun angles.

The MAS optics are set up to emulate, in reverse, the illumination of the starshade by sunlight. A general schematic and image is shown in Fig. 3. To summarize, a collimated, calibrated 635-nm laser illuminates the terminal radius of an optical edge coupon that scatters light into a detector. The coupon and laser light can be rotated about 2 degrees of freedom to simulate the Sun moving to different positions relative to the starshade. Whereas in space the starshade edges are illuminated by the 30-arc min wide Sun and observed over the infinitesimal angle of the distant telescope aperture, for convenience in the laboratory the illumination is with a collimated laser beam and the detector integrates the scatter over a 30-arc min wide acceptance cone equivalent to the solar disk size. The signal scattered by the illuminated edge is compared to the signal of the illuminating beam shining directly into the camera with the edge removed. The ratio is the fractional scatter at the $30-\mathrm{cm}$ distance of the detector. The SDF is the fractional scatter over the range of solar angles and edge orientations, scaled to a standard distance of $1 \mathrm{~m}$. Every point in the SDF (Fig. 4) corresponds to the fractional scatter at the angle of the Sun from the starshade normal (vertical axis) and a position angle of the optical edge relative to the Sun and telescope (horizontal axis). Specular reflection is at $0^{\circ}$ on the horizontal axis. The data from the SDF are read by the solar glint code in the SISTER simulations.

We note that, unlike a bi-directional reflectance distribution function, the scatter from the starshade is always observed at $90^{\circ}$ from the starshade axis (with a small inconsequential tolerance for wobble as the starshade spins). Thus, the SDF only considers the range of incident Sun angles, with the observer always at $90^{\circ}$ to the reference plane.

The repeatability of the MAS over several months is the largest contributor to the uncertainty in the estimated level of glint. Measurements of the scatter of an AM coupon between February and October 2019 had a standard deviation of $10 \%$. It is possible that some of this is due to changes in both the position of the measurement along the edge, as well as contaminants. However, we are not able to separate those effects from the instrument itself and therefore use the measured repeatability of $10 \%$ in our estimation of the MAS precision.

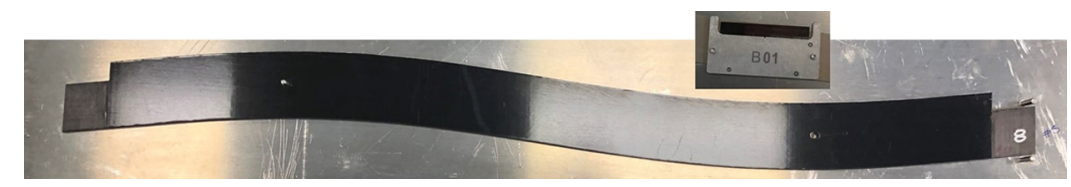

Fig. 2 A 500-mm-long optical edge segment. The AM edge is sandwiched between graphite composite substrates. Inset: an AM coupon mounted in its holder.

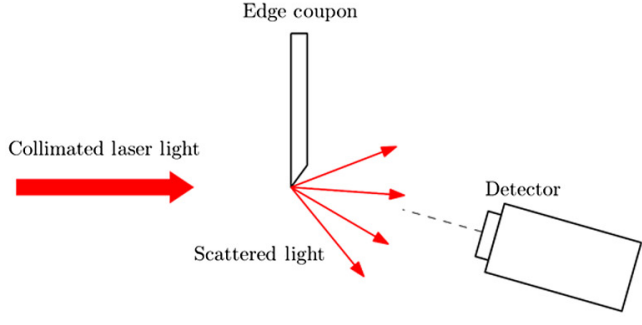

(a)

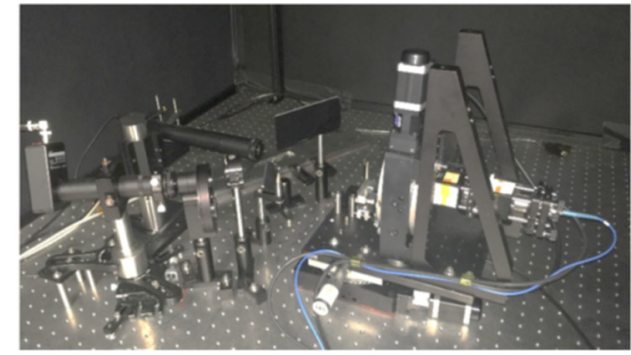

(b)

Fig. 3 (a) Simplified schematic of the MAS and (b) image of assembled instrument. 


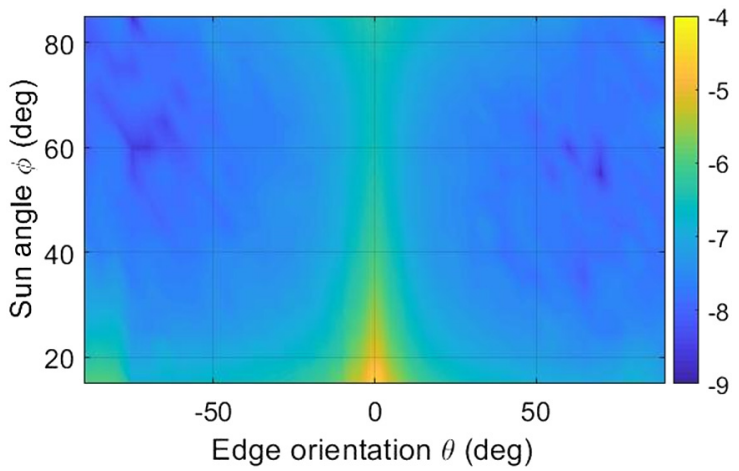

Fig. 4 Log-intensity display of the fractional intensity SDF, with edge orientation relative to the Sun on the horizontal axis and solar angle with respect to the starshade normal on the vertical axis. This SDF is formed from the average of 13 AM edge coupons (see Sec. 6). The intensity units are scaled to show the log of the fractional incident solar energy per meter of edge length, observed from a distance of $1 \mathrm{~m}$.

\subsection{Single-Angle Scatterometer}

The SAS measures scatter along the length of an edge up to 1-m long but only over a fixed cone angle. Because the SAS has no means of measuring the unscattered beam, it has no absolute scatter calibration; it is used to determine the ratio of one scattering edge relative to another, e.g., etched coupons relative to razor blades and edge segments relative to coupons. The segment-tocoupon scatter ratio (SCSR) is the primary product that is used to link the MAS coupon measurements to SAS segment measurements, which together form the basis of the solar glint imaging simulation.

The optics of the instrument are composed of a 635-nm laser launcher, an alignment detector, and a camera tube. A test article is rigidly mounted while the instrument is fixed to two translation stages and one rotational stage, such that the camera can follow the edge and orient itself perpendicular to it. A final vertical stage is attached to the camera itself for focusing. As with the MAS, a laser illuminates the terminal radius and scatters light into the camera. A schematic of the major components and a picture of the device are shown in Fig. 5. Unlike the MAS, camera images the edge scatter so that the local scatter characteristics can be studied. Figure 6 shows a typical image. All detectors and the camera have narrow bandpass filters to remove the majority of room light. More detail on each component and the operation of the instrument follows.

A single-mode optical fiber and laser collimator expand a laser beam to a diameter of $10 \mathrm{~mm}$. The beam is linearly polarized such that roughly equal power is produced in both S- and Ppolarizations at the output. The polarized beam passes through a 10:90 (R:T) non-polarizing

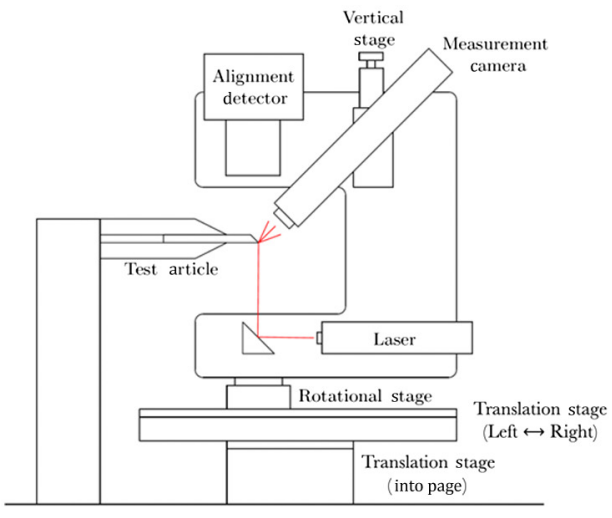

(a)

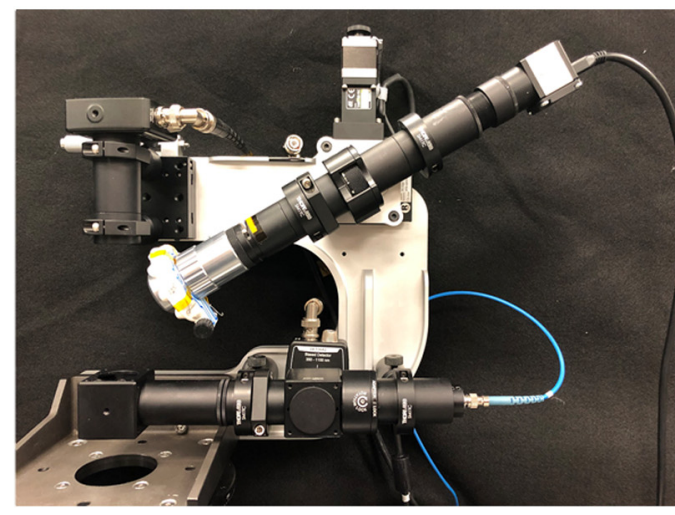

(b)

Fig. 5 (a) Schematic diagram of fixed angle scatterometer setup and (b) image of assembled instrument. 


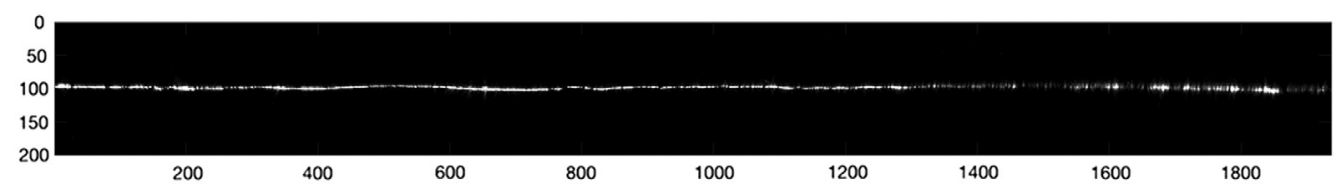

Fig. 6 SAS image of scattered light from an optical edge segment. Axis units are in camera pixels. The field of view is $\sim 1 \mathrm{~mm}$.

beamsplitter. The reflected light is then measured with a silicon detector to monitor power fluctuations. The majority of the light passes through the beamsplitter and is reflected toward the test article, illuminating the terminal edge from below.

An alignment detector is positioned above the sample and consists of a collecting lens, a neutral density (ND) filter, a bandpass filter, and a silicon detector. The alignment detector is used to ensure that the laser is centered on the edge.

The camera tube consists of a long working distance $10 \times$ microscope objective with an acceptance angle of $\sim 30^{\circ}$, a focusing lens, linear polarizer, bandpass filter, and a high-resolution CMOS monochrome camera. The scattered light from the terminal edge enters the objective and is focused through the filters onto the camera. The camera is able to image roughly $1 \mathrm{~mm}$ of edge at a time. At each measurement location, light is gathered over that length of edge from a range of incident angles between $45^{\circ}$ and $75^{\circ}$ from the edge normal.

The translation stages consist of a 1-m longitudinal stage, a perpendicular 200-mm stage for lateral movement, a rotation stage, and a small $15-\mathrm{mm}$ vertical stage. The optics are held to the rotation stage with an aluminum bracket. The vertical stage is also mounted to this bracket and holds only the camera tube, as the other optics do not need a vertical range of motion.

The instrument is aligned to a test article by manually finding the corners of the terminal edge using a reticle on the camera output. Using this process, the instrument can be repeatably positioned within $50 \mu \mathrm{m}$. The position of the edge is then interpolated by the software, which automatically re-centers and refocuses the camera on the terminal edge at each measurement location.

A sample set of SAS output data is given in Fig. 7. Each data point on the plot corresponds to a separate SAS measurement at $1 \mathrm{~mm}$ spacing along the edge. Each measurement is the integral of the light across the camera, an example of which was shown in Fig. 6 . The vertical axis units are the fractional scatter at a distance of $1 \mathrm{~m}$, for a 1-m length of edge, at a wavelength of $635 \mathrm{~nm}$.

The fractional intensity scale in Fig. 7 was determined by comparing the SAS segment scatter to the mean SAS scatter from a set of coupons (the SCSR). The coupons were also measured on the MAS where their absolute scatter characteristics were determined. The process of linking the SAS and MAS measurements is described in Sec. 2.3.

To verify SAS repeatability, a single edge coupon was measured over the central $90 \%$ of its length on six separate occasions during the six-week course of the data collection. The resulting repeatability of the mean scatter had a standard deviation of $0.9 \%$. In practice, reference coupons are measured regularly to ensure that the instrument calibration is not drifting.

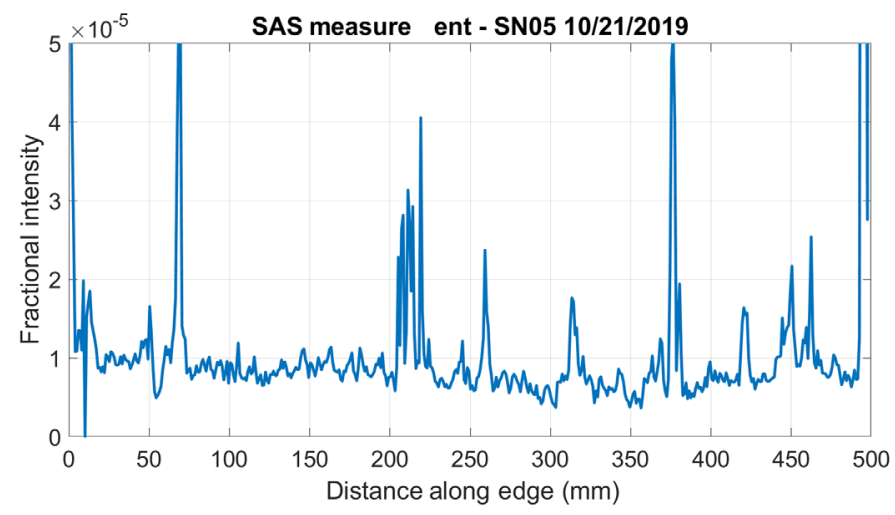

Fig. 7 Sample data taken with the SAS instrument. 


\subsection{Relating the SAS to the MAS}

The MAS measures the absolute SDF of edge coupons at angles $(\theta, \phi)$, spanning the full range of incident solar angles and edge orientations necessary to compute the solar glint lobe pattern. The SAS measures the integrated scatter of edge coupons relative to edge segments over a $15^{\circ}$ radius of the SDF centered at a Sun angle of $60^{\circ}$ and specular edge orientation (Fig. 8). The link between the SAS segment measurements and the MAS coupon measurements is the circular region of the SDF. For a given coupon edge, the ratio of the light within the SAS acceptance cone to the light within the full MAS rectangle is termed as the selection ratio, $\rho$. A perfectly specular edge will only reflect light at $\theta=0^{\circ}$, leading to $\rho=1$. Razor blades are highly specular reflectors and we find $\rho=0.95$. AM blades are more diffuse and the selection ratio based on measurements of 13 coupons drops to $\rho=0.6$ with a standard error of the mean (SEM) of 0.029 (4.8\%). The SEM of $\rho$ is one of contributing error terms in the glint lobe estimation accuracy detailed in Sec. 6 .

With measurements of the relationships between the cone and the full range of edge angles, and between the segments and the coupons, the data are combined as shown in Fig. 9 to compute the SDF used by the imaging software. The MAS coupon measurements indicate the fraction, $\rho$, of the total scattered energy that the SAS is measuring. The measurement of coupons by both the SAS and MAS provides the relative calibration of the two instruments. The SAS

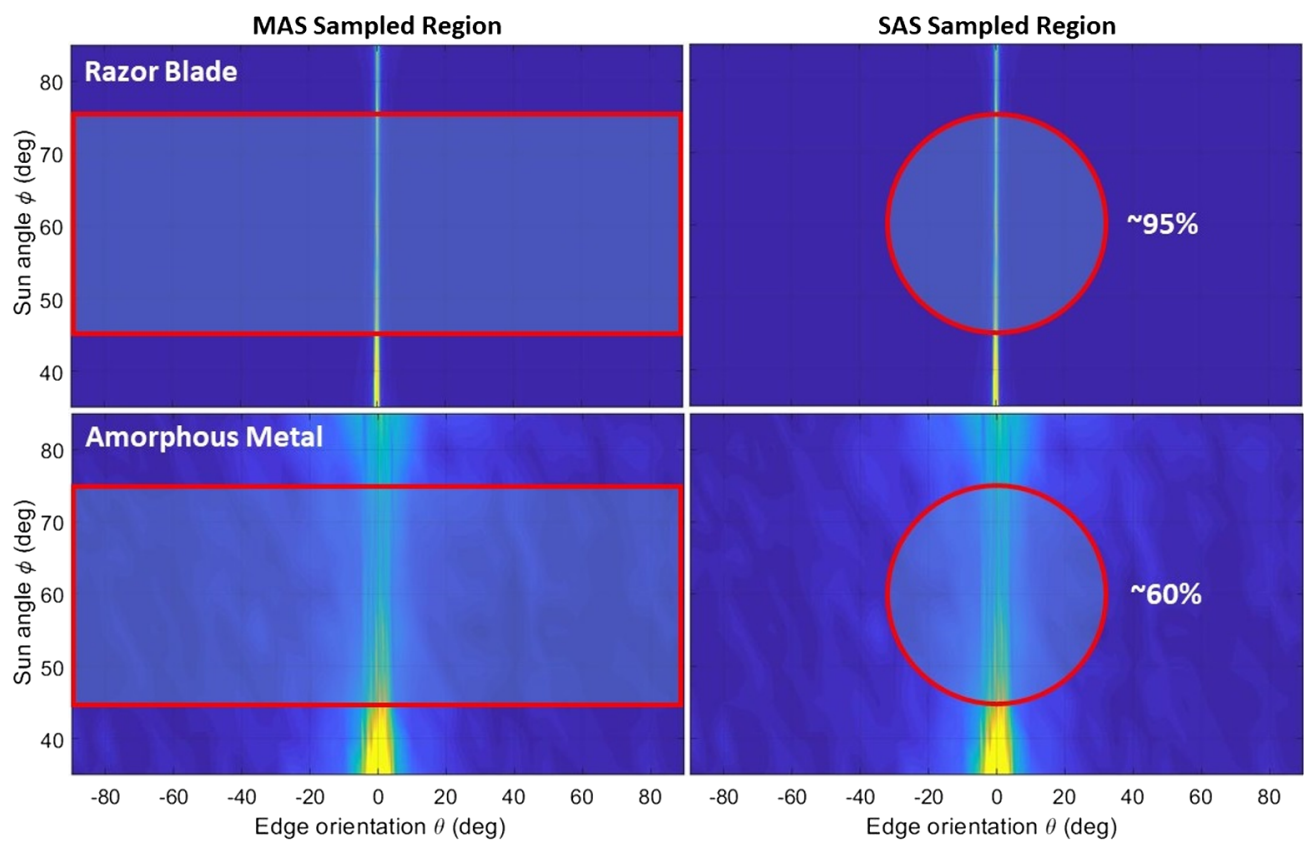

Fig. 8 SDF, plotted on a linear scale, indicating the difference between a highly specular razor blade and a more diffuse AM edge between the two scatterometer testbeds. The circle represents the SAS acceptance cone. The ratio of light captured by the SAS to light at all edge orientations over the same range of solar angles is shown to the right of the acceptance cone circle.

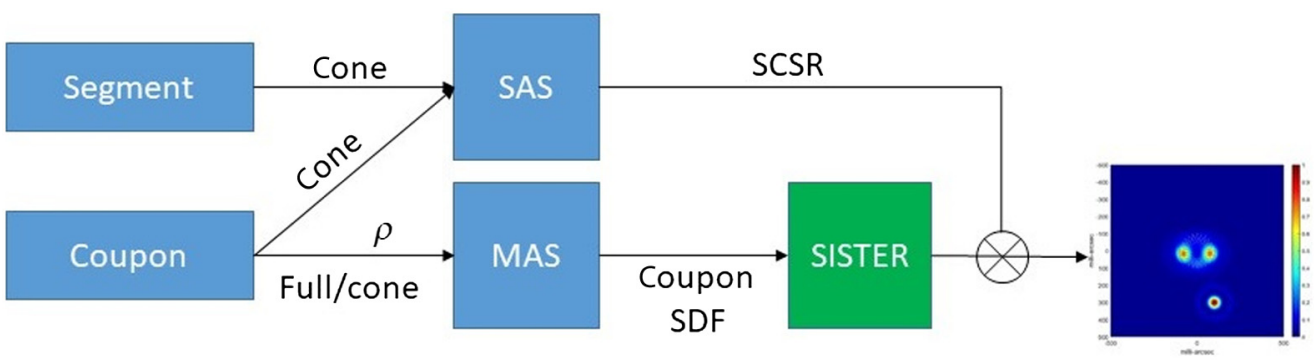

Fig. 9 Flowchart of measurements and analysis. Solar glint is computed from the average coupon scatter heatmap multiplied by SCSR. 
measurements of the two instrument, and the SAS measurements of the segments and coupons yields the SCSR. The output product is the average SDF of the coupons multiplied by the SCSR; this then represents an estimate of the segment SDF.

\section{Modeling Solar Glint Lobes}

A small fraction of sunlight will glint from the edge of the starshade into the telescope. Even at a distance of tens of thousands of kilometers, solar glint from the metallic, razor-sharp edges is the brightest source of instrument background. ${ }^{15}$ For the SRM starshade, roughly $130 \mathrm{~m}$ of edge contributes to the glint, of which $\sim 10 \mathrm{~m}$ has a strong specular component that samples the region $\theta \sim 0$ in Figs. 4 and 8.

The JPL-developed Starshade Imaging Simulation Toolkit for Exoplanet Reconnaissance (SISTER) is used to compute the solar glint lobes. ${ }^{8}$ SISTER reads the two orthogonal polarization SDFs generated by the MAS. It also reads in the locus of points constituting the outline of the starshade. Given the position of the Sun relative to the starshade and telescope, it calculates the relative Sun-to-edge angle and determines where on the SDF to sample the scatter. The edges are categorized as "leading" and "trailing": leading edges are defined as having the Sunstarshade-telescope angle less than $180^{\circ}$ such that light can both reflect and diffract into the telescope. Trailing edges require the light to diffract toward the telescope, with no possibility of reflection. The code assumes that the trailing edges are shadowed from structure of the edge assembly or from self-shadowing due to the edge bevel profile and do not contribute to the glint. The code also assumes that the inter-petal gaps, where the edges are within a few centimeters of each other, are shadowed using small structures on the Sun-facing side and do not contribute to the glint. The contributions of all illuminated edge segments are convolved with the telescope point spread function at each wavelength being simulated (Fig. 10).

To validate the accuracy of the SISTER calculations, we generated a test starshade with a triangular shape having a 1m-long edge oriented for specular reflection of sunlight. We assumed that the edge had a negligible terminal radius of curvature so that only diffraction was present. We applied the Sommerfeld diffraction equations for the "S" (surface parallel) polarization and generated an SDF for a specular edge. This SDF was substituted for the experimental measurements. With the Sun positioned at $\phi=80^{\circ}\left(10^{\circ}\right.$ behind the plane of the starshade) SISTER then calculated a glint lobe.

We calibrated the magnitude of the glint lobe by modeling a planet of known magnitude. We used SISTER to generate a planet well to the side of the starshade (so as not to be attenuated by the starshade). We chose the host star to be the Sun at a distance of $10 \mathrm{pc}(V=4.83)$ and chose the planet to have a flux ratio of $8.55 \times 10^{-9}$ (delta mag $\left.=20.17\right)$. This created planet with a
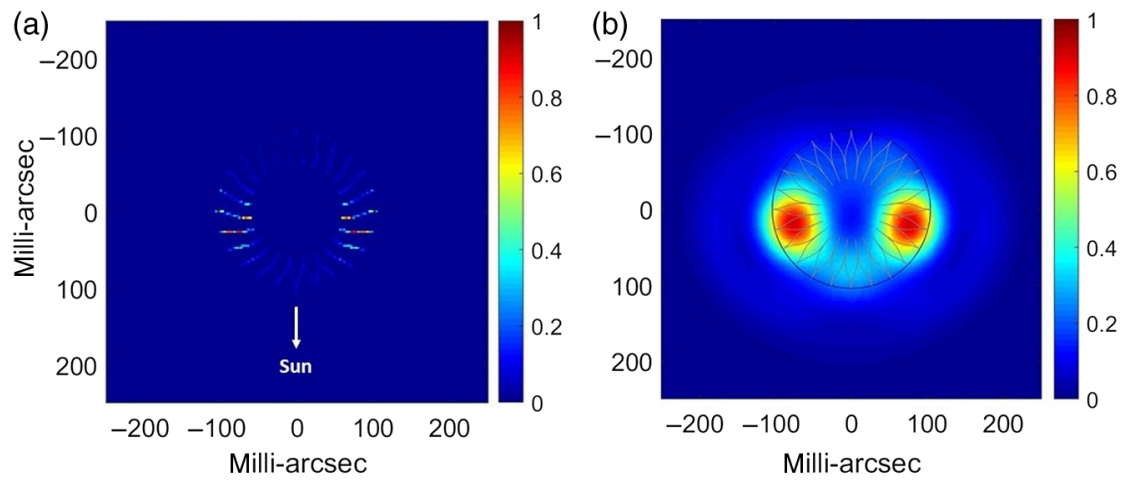

Fig. 10 (a) The normalized solar glint pattern for the SRM starshade at a distance of $25.7 \mathrm{Mm}$. The Sun is behind and below the starshade, $67^{\circ}$ from the starshade normal. We have used the measured SDF for AM edges shown in Fig. 4. At the innermost parts of the petals $(r<7.5 \mathrm{~m})$ where the adjacent edges are close together, structure behind the starshade blocks sunlight from illuminating the edge. (b) The glint lobe pattern for a bandpass of 615 to $800 \mathrm{~nm}$ as seen by the Roman Space Telescope. The flux is normalized to the peak flux of the image of a $V=25$ A0 V star. The circle around the starshade is the IWA. 
visual magnitude $V=25$. Comparing the planet to a glint lobe, we found that the integrated light of the glint lobe was $V=24.65$.

We then compared this to the analytical calculation for a 1-m edge at the distance of the starshade (simply carried out on a spreadsheet), and assuming only that the Sun had a visual magnitude of -26.76 , the value used in SISTER, obtained the predicted glint result of $V=24.64$. We thus conclude that errors in the SISTER glint code are $0.01 \mathrm{mag}$ or $1 \%$.

The glint is concentrated inward of the starshade's outer tips, as shown in Fig. 10. In this example, which is based upon the measured scatter from segments described in Sec. 4, the Sun is at $\phi=67^{\circ}\left(23^{\circ}\right.$ behind the plane of the page). Those parts of the starshade edge that are horizontal in the picture contribute most of the glint. The result is a two-lobed pattern, with each lobe only partially resolved at the resolution of the telescope.

\section{Optical Edges}

\subsection{Design Overview}

The optical edge precisely defines the perimeter of each petal. It is not continuous but is made up of segments approximately 1-m long that are bonded to the structural edge of the petal, as depicted in Fig. 11. The structural edge is a continuous piece of CFRP defining the perimeter of the petal primary structure.

A cross section of an optical edge bonded to the petal is shown in Fig. 12(a). The substrate CFRP layup matches that of the structural edge. It provides structural support to a $38-\mu \mathrm{m}$-thick AM foil. The edge of the foil defines the terminal edge of the segment and therefore the petal. The components are bonded together with EA9394 epoxy, chosen for its relatively high strength across a broad and relevant temperature range, creep resistance, long pot life, and history of use in flight programs. Two bond operations are necessary to assemble an optical edge: first, the AM is bonded to the CFRP substrate to produce a segment, then that segment is bonded to the structural edge. The substrate is bonded to within $0.64 \mathrm{~mm}$ of the terminal edge to support the thin foil, whereas the structural edge is offset $\sim 12 \mathrm{~mm}$ inboard to prevent sunlight from illuminating the telescope side of the starshade. All bond line thicknesses are controlled using 0.005-in.-diameter glass beads. A detail of the terminal edge is provided in Fig. 12(b).

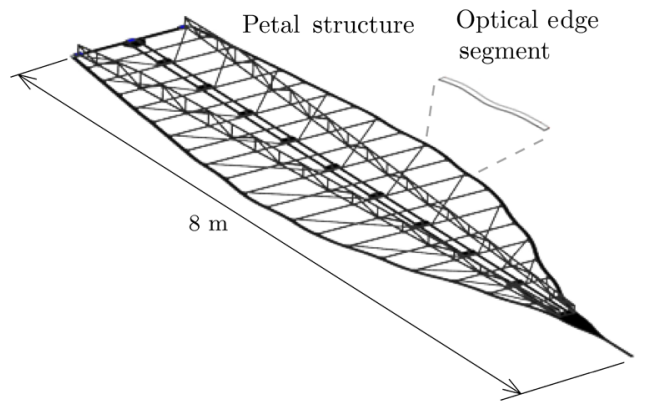

Fig. 11 Petal mechanical structure with placement of optical edge.

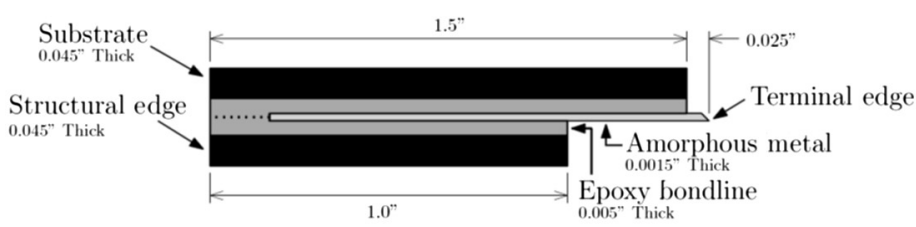

(a)

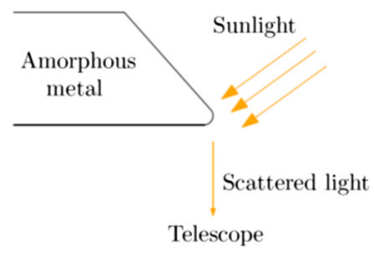

(b)

Fig. 12 (a) Cross section of optical edge flight and prototype designs, see section A-A in Fig. 13 and (b) detail of terminal edge showing the orientation with respect to the Sun and telescope. 
$\mathrm{AM}$ is chosen to form the terminal edge due to the results of a previously performed trade study. ${ }^{16}$ The typically large grain structure found in most off the shelf metals and alloys creates large, irregular etched feature sizes, resulting in more scattered light reaching the telescope. In contrast, the glassy molecular structure of AMs results in a relatively smooth and thus sharp bevel well suited for this application. The specific alloy used is known by the trade name MBF23 and is manufactured by Metglas Inc. The alloy constituents include $60.5 \% \mathrm{Fe}$ and $30 \% \mathrm{Ni}$ by mass percent, along with small amounts of $\mathrm{Cr}, \mathrm{B}$, and $\mathrm{Si}$.

\subsection{Test Articles}

Two sets of test articles were fabricated: assembled 500-mm segments and standalone 50-mm AM coupons. The segments incorporate etched AM bonded between two pieces of carbon fiber as depicted in Fig. 12. The segments are too large to be measured in the MAS. Therefore, coupons manufactured in the same lot as the segments were used to characterize the performance of bare AM over all potential Sun angles as well as to establish the SCSR, as described in Secs. 2.2 and 2.3.

\subsubsection{Segments}

A total of six prototype segments were constructed as part of the MS3 effort. The segments were subjected to a full battery of environmental tests. The segments are full scale in cross section and, at 500-mm long, are approximately half scale in length compared to the flight design. This section describes the design and manufacturing process for segments in detail.

The design of the segment test articles is derived directly from the flight design described in Sec. 4.1. The cross section is identical to that shown in Fig. 12, whereas the in-plane shape is a 500-mm-long sinusoid with a peak-to-peak amplitude of $20 \mathrm{~mm}$, as shown in Fig. 13. This shape was chosen because it is consistent with the expected curvature of the petal and enables easy detection of in-plane shape changes with traditional data analysis tools. Although the length is about half that of full scale, all other dimensions (e.g., thickness and width) are full scale to preserve critical dimensions for manufacture and test. Additionally, all components are flight grade materials. The structural edge is included in the assembly and extends $25 \mathrm{~mm}$ beyond the ends of the substrate to mimic the continuous nature of the petal structure.

The purpose of constructing these segments was to demonstrate scatter performance before and after environmental tests, and separately to verify in-plane shape performance.

Construction of the optical edge segments is a multi-step process. The AM was photochemically etched using a commercial process to form the precision edge while the CFRP structural components were routed from fabricated panels. The edge components were then bonded together as described above to ensure the in-plane shape of the foil was preserved and the terminal edge was not damaged.

\subsubsection{Coupons}

The coupons were etched in the same lot as the segment AM and are 25-mm wide $\times 50$-mm long rectangles with straight edges (i.e., they do not have the curvature of the petal). Since the coupons were etched at the same time as the segments, they have the same precise beveled edge and sharp terminal radius. They are small enough to be measured by the MAS. The performance of the coupons was checked before assembly of the segments to establish confidence that the segments would meet scatter requirements. Special handling processes were established to ensure

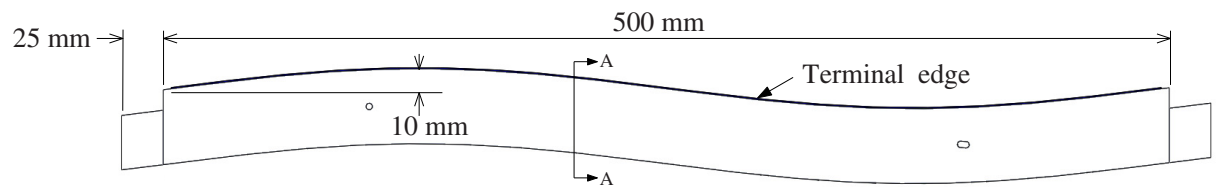

Fig. 13 Layout of an as-built optical edge segment. The cross section A-A is given in Fig. 12. 
that the terminal edge was not damaged before testing. Each coupon was clamped in a two-part aluminum container that protected the terminal edge (Fig. 2) but also allowed for scatter testing and imaging with a scanning electron microscope.

\section{Stow, Release, and Thermal Cycling Tests}

The scattered light performance of the segments was characterized before and after a series of thermal and deployment cycles meant to represent the pre- and post-launch, and on-orbit stressing environments.

The relevant criterion in defining the stressing environments for the optical edge was whether or not the environment could have a detrimental effect on the scatter performance of the terminal edge. In other words, the most critical environments are those that could change the physical attributes of the sharp terminal edge that scatters sunlight. Two environmental tests were deemed to fit this criterion: (1) stow and release cycles at ambient temperature and pressure and (2) thermal cycles in the deployed configuration.

Stow and release cycles are performed at various points throughout a flight test campaign. Although the strain in the AM will be small due to its proximity to the petal's neutral axis, there is an unknown and difficult-to-model potential for the furling strain to manifest itself as a physical change of the very thin and sharp terminal edge. This test captures the actual furling strain environment that the edges will see during the flight test campaign, and therefore any change in scatter performance as well.

Thermal cycling in the deployed configuration serves two purposes. First, it demonstrates whether or not exposure to the expected flight temperature extremes could result in sub-micron level changes to the very thin and sharp terminal edge of the AM that affect scattered light performance. Second, because the AM is bonded to low coefficient of thermal expansion (CTE) CFRP, there is thermal strain between the AM and CFRP that could manifest itself in small ripples or waviness to the edge; this test will demonstrate the extent to which the thermal strain affects solar scatter performance.

Stowed thermal cycles are important for determining the mechanical limits of the bond between the AM and CFRP structure in the space environment; therefore, thermal cycling in the stowed configuration was performed on edge assemblies at various temperature limits. Scatter performance was measured after the stowed thermal cycle environment to confirm the assumption that it was not a stressing environment.

\subsection{Stow and Deploy Cycles}

To stow a starshade, each petal is wrapped in a spiral pattern around the 2.25 -m-diameter folded perimeter truss, although the spirally wrapped petal of the flight article takes a slightly larger curvature. To conservatively bound the worst bending case, the test articles were stowed to a 2.25-m diameter. Although a starshade is only deployed once in space, the flight system is expected to go through a series of on-the-ground test deployments. For the purposes of this study, 10 cycles were determined to encompass the likely number of ground deployments a petal would experience.

The desired radius of curvature was achieved with a fixed-displacement four-point bending fixture. The design of the fixture was driven by the desire to use the same fixture for stowed thermal testing, requiring it to fit inside an oven and to be easily transportable. A schematic and image of the bending fixture are shown in Fig. 14.

The desired radius of curvature was achieved with a fixed displacement four-point bending fixture. The design of the fixture was driven by the desire to use the same fixture for stowed thermal testing, requiring it to fit inside an oven and to be easily transportable. A schematic and image of the bending fixture are shown in Fig. 14. The fixture was assembled using T-slotted aluminum framing and other off the shelf components. The top half of the fixture was constrained on two 19-mm-diameter bushings. Displacement, and therefore curvature, was set using three fine pitched 1/4-80 set screws on the ends of the fixture. The inner pins were set to be a distance of $L / 4$ from the outer pins, where $L$ is the distance between the two outer pins. 


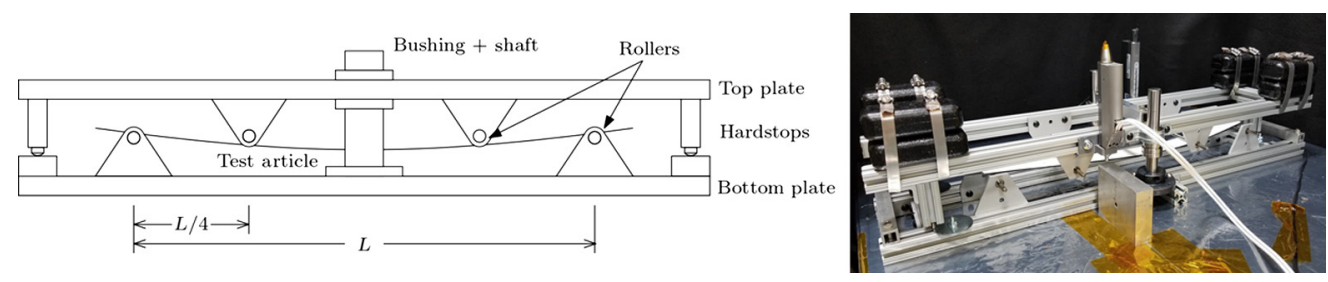

Fig. 14 (a) Schematic diagram of the four-point bending fixture and (b) fixture in use including loading weights, micrometers, and an installed edge (white).

This configuration was chosen because it provides a sufficiently long region $(250 \mathrm{~mm})$ in the middle of the test article where theoretically constant curvature and zero shear are applied while also avoiding excessive shear in the outer regions compared to the expected flight values. The displacement-driven configuration is more representative of the boundary conditions of the flight hardware system and is more deterministic than a load-driven configuration. This in turn improves the overall ability to correlate our test configuration to our model.

The vertical displacement was set to achieve the desired radius of curvature of $1.125 \mathrm{~m}$. Simple textbook equations and estimated bulk material properties were used to calculate the bending moment and shear in the test specimens under four-point bending:

$$
M_{\max }=F \cdot x \quad V_{\max }=F,
$$

where $F$ is the load applied to each pin and $x=\frac{L}{4}$. This estimation showed that the test articles should experience a bending moment of about $3.8 \mathrm{~N} \cdot \mathrm{m}$ between the two inner pins and a shear load of $32.5 \mathrm{~N}$ in the outer regions. To verify that these loads are similar to what would be seen in a flight system, a petal-level finite element model of the stowed configuration was analyzed. Shear and moment loads were checked throughout the deployment sequence in addition to the fully stowed state. The model showed a maximum shear load of $11.1 \mathrm{~N}$ and maximum bending moment of $3.6 \mathrm{~N} \cdot \mathrm{m}$ in the optical edge, the maximum value being defined as the maximum average stress across the cross section of the edge. The test article conditions are considered to be representative and conservative for the flight petal.

After a test article was placed in the fixture, the raising and lowering rate was controlled with two motorized micrometers operating in tandem and programmed to move at a velocity of $0.5 \mathrm{~mm} / \mathrm{s}$, a sufficiently slow rate to simulate expected ground handling and flight deployment speeds.

The starshade petals can be bent in one of two orientations depending on where they are attached on the truss. Since the neutral axis of the optical edge assembly is slightly offset toward the substrate side, the metal foil can be put into either compression or tension depending on the bending direction. Therefore, half of the assemblies were tested with the AM in compression and the other half in tension.

\subsection{Deployed Thermal Cycle}

The stressing condition for an optical edge in the deployed configuration is thermal loading resulting from the mismatch in CTE between the AM and CFRP components. The CFRP has a near-zero CTE while the AM has a measured value of $8.4 \mathrm{ppm} /{ }^{\circ} \mathrm{C}$.

Deployed thermal cycling occurred in a nitrogen purged chamber at JPL. The edges were placed on a flat plate in an unconstrained state. A starshade system-level thermal analysis was used to determine test temperatures. The finite element model is detailed and incorporates all major components of the starshade, including petal structure, optical shield, and other blanketing. It assumes all CFRP surfaces, except the optical edges, are covered in single-layer insulation on the Sun side and a three-layer optical shield on the telescope side. The Sun-facing layer consists of a single layer of Kapton, doped with silicon on the Sun side and aluminized on the anti-Sun side for favorable thermal properties. The two remaining layers are composed of black Kapton XC for its low light transmissivity property. 
The analysis assumed that the starshade would spin at 1/3 RPM. Because the spinning of the starshade equalizes the temperature of all like components on the starshade, the primary variable in determining the maximum and minimum temperature of the optical edge is the angle of the Sun relative to the starshade. During science operations, the Sun will fall between $\sim 40^{\circ}$ and $83^{\circ}$ relative to starshade normal. In addition, the starshade must also be able to survive a Sun angle of $0^{\circ}$ as the starshade re-orients and maneuvers into position at next target star.

The maximum temperature of $80^{\circ} \mathrm{C}$ occurs when the starshade is normal to the Sun, $\phi=0^{\circ}$, and the minimum temperature of $-96^{\circ} \mathrm{C}$ occurs with the Sun to the side of the starshade at $\phi=83^{\circ}$.

To provide temperature margin compared to the model predictions, we tested the edge assemblies over a temperature range of $+105^{\circ} \mathrm{C}$ to $-125^{\circ} \mathrm{C}$. Note that $\phi=180^{\circ}$ (Sun shining on the telescope side of the starshade), while not a mission design case, was also analyzed and found to have nearly identical results to the $0^{\circ}$ case.

The number of thermal cycles in the deployed configuration for SRM is expected to be under 40. This is based on the number of target star re-orientations, a maneuver that results in the starshade changing orientation with respect to the Sun, and thus cycling the temperature of the starshade. For this test, a minimum of 25 cycles was performed on all edges, and 50 cycles on one edge assembly, as well as on the edge coupons.

\section{Optical Scatter Test Results}

Here, we summarize the results of scatter testing of a set of 13 coupons and 4 segments. Five of the coupons were manufactured concurrently with the segments, whereas the rest were manufactured using the same process weeks later. We observed no statistically significant difference in performance between the two lots and so treat them as a single set of coupons throughout this document.

Three of the four segments were measured before and after thermal environmental testing (the fourth only went through a partial set of tests), and we found that the mean level of scatter after environmental testing was $0.99 \pm 0.15$ relative to the pre-environmental measurements made several weeks earlier. Figure 15 shows an example of the pre- and post-environment SAS measurements of one segment. Based on these measurements, we conclude that the environmental testing showed no significant change in the optical performance of the edges.

\subsection{Experimental Error Budget}

We estimate that the our glint lobe brightness predictions are accurate to $\pm 17 \% 1-\sigma$. This is based on measurements of the instrument repeatability and accuracy, small sample size limitations of the coupons and segments, and the accuracy of the imaging code. These quantities are identified in Table 2. All values listed in the table and in the discussion below are the measured

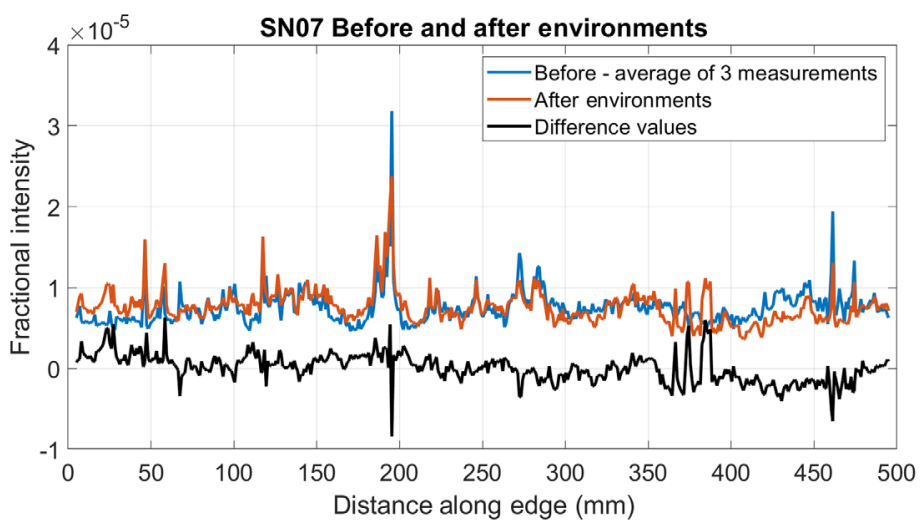

Fig. 15 Pre- and post-environmental measurements of a 50-mm-long edge segment. Measurements are normalized to a 1-m edge observed at a distance of $1 \mathrm{~m}$. 
Table 2 Sources of error in estimating solar glint lobe brightness.

\begin{tabular}{|c|c|c|}
\hline Parameter & $\begin{array}{c}\text { Error } \\
(1-\sigma)\end{array}$ & Notes \\
\hline \multicolumn{3}{|l|}{ Instrument } \\
\hline MAS repeatability & $10 \%$ & $\begin{array}{l}\text { Standard deviation of instrument repeatability on a coupon } \\
\text { (3-mm spot) }\end{array}$ \\
\hline MAS scatter calibration & $5 \%$ & Knowledge of open beam ND filter OD \\
\hline MAS length scale & $2 \%$ & Accuracy of distance from coupon edge to aperture $(300 \mathrm{~mm})$ \\
\hline SAS repeatability & $0.9 \%$ & $\begin{array}{l}\text { Standard deviation of instrument repeatability on a coupon } \\
\text { (3-mm length) }\end{array}$ \\
\hline \multicolumn{3}{|l|}{ Coupons } \\
\hline Mean coupon scatter (MAS) & $7.6 \%$ & $\begin{array}{l}\text { SEM of coupon-to-coupon variability integrated over full range } \\
\text { of angles }\end{array}$ \\
\hline $\begin{array}{l}\text { Mean coupon selection ratio } \rho \\
\text { (MAS) }\end{array}$ & $4.8 \%$ & SEM of the ratio of coupon cone scatter to coupon full scatter \\
\hline Mean coupon scatter (SAS) & $6.7 \%$ & SEM of coupon-to-coupon integrated scatter \\
\hline Mean MAS-to-SAS ratio & $3.8 \%$ & $\begin{array}{l}\text { SEM of relative cone scatter of the same 3-mm spot } \\
\text { measured on each instrument }\end{array}$ \\
\hline \multicolumn{3}{|l|}{ Segments } \\
\hline Mean segment scatter (SAS) & $4.2 \%$ & SEM of segment-to-segment average scatter \\
\hline \multicolumn{3}{|l|}{ Total } \\
\hline Root sum square error & $17.0 \%$ & Estimated $1 \sigma$ error on glint lobe brightness \\
\hline \multicolumn{3}{|l|}{ MUF } \\
\hline Imaging code (SISTER) & $1 \%$ & Consistency between analytical model and SISTER glint lobe \\
\hline \multicolumn{3}{|l|}{ Final uncertainty } \\
\hline MUF $\cdot$ RSS error & $17.1 \%$ & $1 \sigma$ error including model uncertainty factor \\
\hline$\Delta$ mag $95 \%$ confidence & -0.27 & $1.65 \sigma$ \\
\hline
\end{tabular}

standard deviation or SEM, where appropriate, unless otherwise stated. The final $\Delta$ mag confidence of $95 \%$ at $1.65 \sigma$ relies on the assumption that the combination of independent errors forms a normally distributed population.

\subsubsection{Instruments}

The MAS provides the SDFs that are used to calculate the glint lobes. As noted in Sec. 2.1, the long-term (month-to-month) repeatability of the MAS SDF, based on measurements of a razor blade we use as a reference coupon, is $10 \%$. The short-term repeatability over the course of this experiment is much better (of order $2 \%$ to $3 \%$ ) but ultimately the long-term stability limits our knowledge of the absolute scatter from the edges.

The MAS measures the ratio of the scatter at a range of angles to the unscattered beam shining directly into the detector. The optics, apertures, and detectors in these measurements are all common mode, except for a ND filter placed in the direct beam. An error in the measurement of the optical density of the ND filter directly translates to an error in the scatter ratio. Using two independent calibrated optical photometers and taking into consideration experimental alignment precision, we measured the filter and determined that the total optical density is 7.18 with an uncertainty of $\pm 5 \%$. 
The measured scatter ratio is a function of the distance from the coupon edge to the detector, nominally $300 \mathrm{~mm}$. With the defining detector aperture in a holder with a recessed edge, we conservatively estimate our distance measurement error is $3 \mathrm{~mm}(1 \%)$. The scatter ratio in the experiment depends on the distance squared; thus, we report a $2 \%$ error on the length scale in Table 2.

The SAS reports a scatter value with an arbitrary (not absolute) scale. As noted in Sec. 2.2, the stability of the measurements over the course of the experiments was determined to be $0.9 \%$.

\subsubsection{Coupons and segments}

The manufacturing process produces coupons and segments with varying scatter characteristics. Our ability to measure the expected level of scatter and the selection ratio is limited by the finite set of coupons (13) and segments (4). Here, we break down the experimental limitations observed and reported in Table 2.

The MAS measures the coupon scatter over the full range of solar angles and edge orientations (the rectangle in Fig. 8). The mean value over the set of coupons has an SEM of 7.6\%. The MAS also allows us to measure the light within the angular cone sampled by the SAS to relate this to the full range, and the ratio has an SEM of 4.8\%. Likewise, the SAS measures the coupons in its cone with an SEM of 6.7\%. Finally, when we compare the energy in the MAS cone to the energy in the SAS, the SEM is $3.8 \%$. We conservatively treat all of these quantities as independent errors even though some of the variability measured in each instrument is actually spatial variability in the coupons.

The SAS measurements of the mean segment scatter are consistent to SEM $=4.2 \%$. Even though there were only four segments considered (and three for post-environmental evaluation), each of the 50-mm-long segments was measured with a $1-\mathrm{mm}$ resolution, leading to a large set of data for the set of edges.

We measured the central $45 \mathrm{~mm}$ (out of $50 \mathrm{~mm}$ ) of the coupons and the central $400 \mathrm{~mm}$ (out of $500 \mathrm{~mm}$ ) of the segments, which was necessitated because some corners had suffered slight damage, and some segments suffered thermal cycle-induced delamination. The scatter profile seen in Fig. 15 is typical of all segments; there are regions a few millimeters wide that are 2 to 10 times brighter than the nominal scatter level. This may be due to damage from handling or epoxy near the edge. The spikes persist after cleaning, which included the application and removal of First Contact Polymer. ${ }^{17}$ This indicates that the spikes are not related to particulate contamination, which is thoroughly removed by the polymer. Coupons also had some scatter spikes, typically double the nominal scatter level.

The partial delaminations, corner damage, and evidence of epoxy leakage show that improvements are required in our segment design and manufacturing process, as well as our handling procedures. These will be the subject of future work.

Segments were found to be brighter than the coupons. We found the median SCSR was $1.35 \pm 0.09$, compared to a mean value of $1.41 \pm 0.11$. The similarity of the mean and median SCSR values shows that the scatter peaks contribute only $\sim 20 \%$ of the difference between segments and coupons. Since the manufacturing processes for the segments and coupons were identical and concurrent, and since the pre- and post-environmental tests showed no significant difference, we explored the possibility that the segment mounts could be contributing to the increased scatter. We measured a spare set of three unmounted segments that we supported in a fixture that held the segments several millimeters from the edge. We found that these segments were $17 \%$ dimmer than the mounted ones. Thus, about half of the excess segment scatter may be due to the CFRP and epoxy. The remaining difference could be statistical variations from the small data sets.

\subsection{Final Glint Lobe Brightness Uncertainty}

In addition to the instrument, coupon, and segment uncertainties, the analysis and lobe prediction by the SISTER software has been shown to be accurate to $1 \%$ (Sec. 3).

In total, these uncertainties combine to yield an estimated uncertainty of $\sim 17 \%$ in the computation of the glint lobe brightness. Assuming the uncertainties are normally distributed, the 
$95 \%$ confidence level is an uncertainty of $28 \%$, which can be expressed as an observational magnitude uncertainty of $\Delta$ mag $=0.27$. In Sec. 7 , we include this offset to report on the 95\% confidence level in our estimate of the glint lobe brightness.

\section{Magnitude of Solar Glint Lobes}

\subsection{Brightest and Average Photometric Pixels.}

The visual magnitude of the solar glint lobes is summarized in Tables 3-5. The evaluation regions for the lobes are shown in Fig. 16. The magnitude of the integrated light in a lobe (column 2 of the tables) is computed by integrating the glint over half the plane, as shown in Fig. 16(a). Much of this light is at radii smaller than the IWA. Column 3 is the magnitude of the integrated light at and beyond the IWA [Fig. 16(b)] and is a full magnitude fainter. To identify a planet, a photometric aperture or matched filter would be used. In columns 4 and 5, and Figs. 16(c) and 16(d), we have convolved the image with a photometric aperture whose diameter is equal to $\lambda / D$, with $D=2.4 \mathrm{~m}$ for SRM and $4 \mathrm{~m}$ for HabEx, at the middle of each band (488.5 and $710 \mathrm{~nm}$ for the SRM blue and green bands, respectively, and $650 \mathrm{~nm}$ for HabEx). These images represent the signal that is measured in the photometric aperture at any point in the image plane. Column 4 and Fig. 16(c) show the magnitude of the brightest photometric pixel for radii $\geq$ IWA. Column 5 shows the magnitude of the mean brightness calculated at the IWA along the path shown in Fig. 16(d). This value represents the average signature of the glint lobes on the

Table 3 Estimated glint lobe magnitude in Roman Space Telescope Rendezvous 425- to 552-nm band.

\begin{tabular}{lccccc}
\hline \hline & Lobe & $r>$ IWA & IWA phot. & IWA phot. & IWA phot. \\
\hline$\phi$ & mag $^{\mathrm{a}}$ & mag $^{\mathrm{b}}$ & min. mag & Avg. mag & ( $^{\mathrm{c}}$ \\
\hline 53 & 25.8 & 26.8 & 26.7 & 27.6 & 27.3 \\
63 & 25.9 & 26.9 & 26.9 & 27.7 & 27.5 \\
73 & 25.7 & 26.7 & 26.7 & 27.5 & 27.3 \\
83 & 25.2 & 26.2 & 26.2 & 27.0 & 26.7 \\
\hline \hline
\end{tabular}

antegrated magnitude of each lobe. See Fig. 16(a).

'Integrated magnitude of each lobe at angles > IWA. See Fig. 16(b).

${ }^{\mathrm{C} B r i g h t e s t}$ photometric pixel at the IWA. See Fig. 16(c).

${ }^{d}$ Magnitude corresponding to the average flux in photometric apertures at the IWA. See Fig. 16(d).

eSame as column (d) with the magnitude adjusted by -0.27 to account for experimental uncertainty (Table 2 ) at the $95 \%$ confidence contour.

Table 4 Estimated glint lobe magnitude in Roman Space Telescope Rendezvous 615- to 800-nm band.

\begin{tabular}{llcccc}
\hline \hline & Lobe & $r>$ IWA & IWA phot. & IWA phot. & IWA phot. \\
\hline$\phi$ & mag & mag & min. mag & Avg. mag & $95 \%$ conf. \\
\hline 53 & 23.7 & 24.7 & 24.6 & 25.5 & 25.2 \\
63 & 23.8 & 24.9 & 24.8 & 25.6 & 25.4 \\
73 & 23.6 & 24.7 & 24.6 & 25.4 & 25.2 \\
83 & 23.1 & 24.1 & 24.1 & 24.9 & 24.6 \\
\hline \hline
\end{tabular}


Table 5 Estimated glint lobe magnitude for HabEx 300- to 1000-nm band.

\begin{tabular}{llcccc}
\hline \hline & Lobe & $r>$ IWA & IWA phot. & IWA phot. & IWA phot. \\
\hline$\phi$ & mag & mag & min. mag & Avg. mag & $95 \%$ conf. \\
\hline 35 & 25.0 & 26.4 & 26.4 & 27.5 & 27.2 \\
45 & 25.6 & 27.0 & 27.1 & 28.1 & 27.8 \\
55 & 25.9 & 27.3 & 27.4 & 28.4 & 28.1 \\
65 & 26.0 & 27.4 & 27.5 & 28.4 & 28.2 \\
75 & 25.8 & 27.1 & 27.3 & 28.2 & 27.9 \\
85 & 25.2 & 26.6 & 26.7 & 27.6 & 27.3 \\
\hline \hline
\end{tabular}
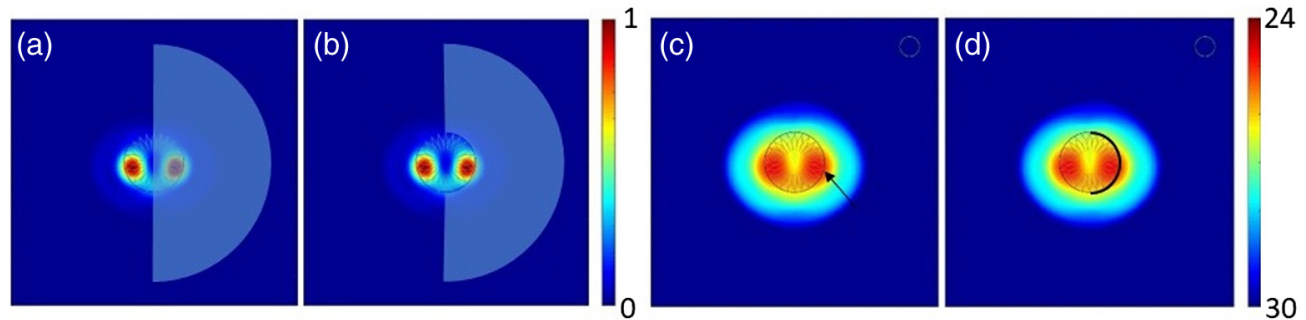

Fig. 16 Evaluation of glint lobe magnitude. SRM lobes are shown and HabEx is similarly analyzed. Each panel spans $500 \times 500$ mas. Panels (a) and (b) are displayed with a linear intensity scale while panels (c) and (d) use a magnitude scale. (a) The lobe intensity is evaluated by integrating over half the plane. (b) The lobe intensity is evaluated only for radii > IWA. (c) The image has been convolved to the resolution of $\lambda / D$. Each point in the image represents the visual magnitude within the photometric aperture. The brightest pixel at the IWA is evaluated. (d) The average value of the scatter at the IWA is evaluated.

detection of planets at the IWA and is typically the value used in evaluations of starshade performance. In column 6, we subtract the measurement uncertainty of 0.27 magnitude from the values in column 5 to account for the $95 \%$ experimental confidence level in the experiment (see Table 2).

We also show how the magnitude of the mean photometric pixel values evolve with working angle for the SRM (Fig. 17). A vertical line marks the IWA radius: 72 mas for the 425- to 552-nm band and 104 mas for the 615- to 820-nm band. The curves show that the glint effect diminishes with increasing distance from the starshade. At a distance of $\lambda / D$ beyond the IWA (112 mas in
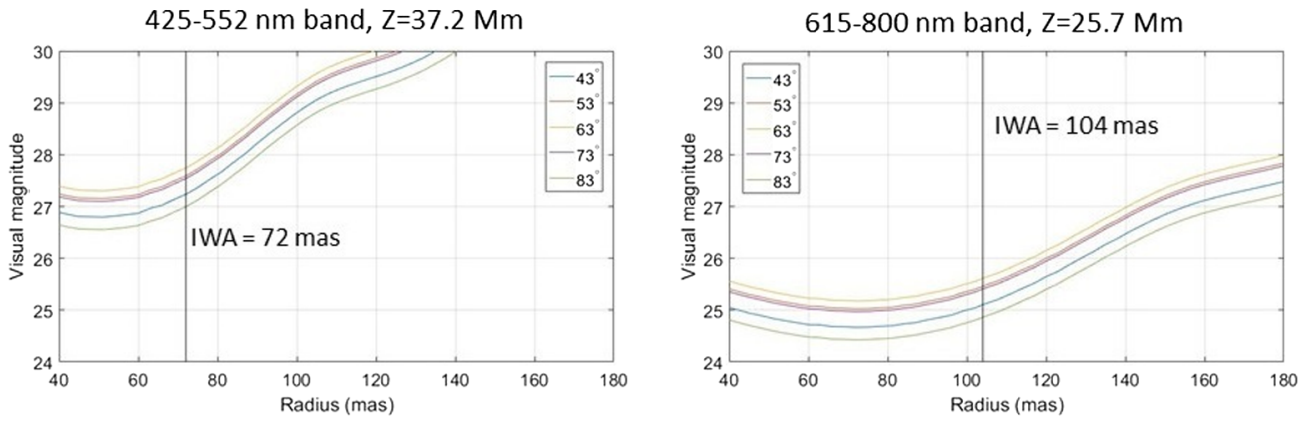

Fig. 17 Magnitude of the Roman Space Telescope average level of illumination per lobe within a photometric aperture as described in Fig. 16. Magnitude values at the IWA are in column 5 of Tables 3 and 4 . 
the 425- to 552-nm band, and 166 mas in the 615- to 800-nm band), the average brightness has dropped by more than 2 magnitudes.

\subsection{Mission Performance Assessment}

The levels of solar glint will significantly impact integration times for a planet appearing near the IWA. In fact, it is several times larger than any other instrument-related background contributor including stellar diffraction due to starshade shape errors, stellar and solar leakage through micrometeoroid holes in the optical shield, and reflection of the Milky Way and other astrophysical objects from the telescope-facing surface. ${ }^{15}$ The SRM's focus on the closest stars, with planets appearing well beyond the IWA, gives ample habitable zone access with minimal interference from the glint lobes. Nonetheless, it is desirable to significantly reduce solar glint to provide the best possible SNR.

Fortunately, there is much of room for improvement. In addition to the potential to produce segments that are close in performance to coupons, the bare metallic edges can be coated with a hybrid interferometric/absorptive coating. The results of our preliminary testing and analysis of these edges show that they improve performance by about an order of magnitude, as reported in the companion paper. ${ }^{9}$

A second mitigating approach is to employ "stealth edges," which is a term we use to describe serrated edges placed where the specular reflection is greatest. ${ }^{2}$ The serration removes the specular component for all but the tips and valleys of the serrated portions and has been shown through MAS measurements to reduce scatter by up to an order of magnitude. The $\sim 1$-mm serration period has no effect on the starlight shadow. However, the stealth edges are not compatible with the baseline approach of spinning the starshade about its axis during an observation. If the starshade does not spin, there will be increased thermal deformation, which in turn would degrade the starlight shadow. This is a trade that is currently under consideration.

\section{Conclusion}

Solar glint from sharp, uncoated metallic edges will appear as partially resolved lobes of magnitude $\sim 25$ (SRM) to $\sim 27$ (HabEx) centered a few meters inboard of the starshade's outer tips. These estimates are based on optical tests of environmentally cycled flight-like optical edges and include formal experimental uncertainties. The tests showed that thermal and deployment cycling caused no significant degradation of performance.

Solar glint from uncoated edges is the brightest source of background light and will significantly increase integration times for Earth-like planets observed at the IWA. To further reduce the scatter, we have explored a promising thin-film hybrid coating described in a companion paper ${ }^{9}$ whose initial assessment indicated an order of magnitude reduction in glint.

The work presented here is an integral part of the Exoplanet Exploration Program Starshade Technology Program. ${ }^{18}$ The starshade technology program has defined a set of eight milestones ${ }^{10}$ to satisfy Technology Readiness level 5, in which a technology is tested at the component or breadboard level in a relevant environment. ${ }^{19}$ The set of milestones includes high contrast optical diffraction and perturbation sensitivity tests (Milestones 1 and $2^{20-22}$ ), optical edge glint (Milestone 3, this work), formation flying (Milestone $4^{23,24}$ ), petal deployment, thermal cycling, and thermal stability (Milestones 5 and 6, under review), and central disk deployment, thermal cycling, and thermal stability (Milestones 7 and $8^{18}$ ). The plan is to continue this work through TRL 6 in preparation for mission opportunities such as SRM and HabEx.

\section{Acknowledgments}

This research was carried out at the Jet Propulsion Laboratory, California Institute of Technology, under a contract with the National Aeronautics and Space Administration (80NM0018D004). We would like to acknowledge the technical assistance of Nicholas Saltarelli and Maxwell Ferguson, and helpful feedback from the Exoplanet Exploration Program Technical Advisory Committee. (C) 2019 California Institute of Technology. Government sponsorship acknowledged. 


\section{References}

1. W. Cash, "Detection of earth-like planets around nearby stars using a petal-shaped occulter," Nature 442, 51-53 (2006).

2. J. Steeves et al., "Development of low-scatter optical edges for starshades," Proc. SPIE 10706, 107065K (2018).

3. Acktar Ltd., 2017, https://www.acktar.com.

4. M. Born and E. Wolf, Principles of Optics, 4th ed., Pergamon Press, New York (1970).

5. D. McKeithen et al., "Modeling the scatter of sunlight from starshade edges," Proc. SPIE 11117, 111171L (2019).

6. S. Seager et al., "The Exo-S probe class starshade mission," Proc. SPIE 9605, 96050W (2015).

7. S. Gaudi et al., "The Habitable Exoplanet Observatory HabEx mission concept study final report," arXiv:2001.06683 (2020).

8. S. R. Hildebrandt et al., "SISTER: starshade imaging simulation toolkit for exoplanet reconnaissance," J. Astron. Telesc. Instrum. Syst. 7(2) (2021).

9. D. McKeithen et al., "Anti-reflection coatings on starshade optical edges for solar glint suppression,” J. Astron. Telesc. Instrum. Syst. 7(2), 021208 (2021).

10. P. Willems, "Starshade to TRL5 (S5) technology development plan," Jet Propulsion Laboratory Publications, 2018, https://exoplanets.nasa.gov/internal_resources/1033.

11. E. Hilgemann et al., "Starshade technology development activity milestone 3: demonstration of solar glint lobe scatter performance," Final Report Report, Exoplanet Exploration Program, 2019, https://exoplanets.nasa.gov/internal_resources/1544/.

12. A. Boss et al., "Exotac report on starshade s5 milestone 3," Review, Exoplanet Exploration Program Technical Advisory Committee, 2020, https://exoplanets.nasa.gov/internal_ resources/1545/.

13. S. Martin et al., "Starshade optical edge modeling, requirements, and laboratory tests," Proc. SPIE 8864, 88641A (2013).

14. E. Hilgemann et al., "Advancements in precision edges for a starshade external occulter," Proc. SPIE 11117, 111170Q (2019).

15. S. Shaklan et al., "Error budgets for the exoplaent starshade (exo-s) probe-class mission study," Proc. SPIE 9605, 96050Z (2015).

16. J. Steeves et al., "Precision optical edges for a starshade external occulter," Proc. SPIE 9912, 991220 (2016).

17. Photonic Cleaning Inc., 2017, https://www.photoniccleaning.com/.

18. "Exoplanet exploration program starshade technology development website," 2019, https:// exoplanets.nasa.gov/exep/technology/starshade/.

19. S. Hirshorn and S. Jeffries, "Final report of the NASA technology readiness assessment (TRA) team," NASA Publication, 2016, https://ntrs.nasa.gov/archive/nasa/casi.ntrs.nasa .gov/20170005794.pdf.

20. A. Harness et al., "Optical verification experiments of sub-scale starshades," J. Astron. Telesc. Instrum. Syst. 7(2), 021207 (2021).

21. A. Harness et al., "Starshade technology development activity Milestone 1A: demonstration of high contrast in monochromatic light at a flight-like Fresnel number," Jet Propulsion Laboratory Publications, 2019, https://exoplanets.nasa.gov/internal_resources/1210.

22. A. Harness et al., "Starshade technology development activity Milestone 1B: demonstration of high contrast in broadband light at a flight-like Fresnel number," Jet Propulsion Laboratory Publications, 2019, https://exoplanets.nasa.gov/internal_resources/1211.

23. M. Bottom et al., "Starshade formation flying I: optical sensing," J. Astron. Telesc. Instrum. Syst. 6, 015003 (2020).

24. T. Flinois et al., "Starshade formation flying II: formation control," J. Astron. Telesc. Instrum. Syst. 6, 029001 (2020).

Stuart Shaklan is the supervisor of the High Contrast Imaging Group in the Optics Section of the Jet Propulsion Laboratory (JPL). He received his $\mathrm{PhD}$ in optics from the University of Arizona in 1989 and has been with JPL since 1991. 
Evan Hilgemann is a mechanical engineer in the Technology Infusion Group at the JPL. He received a BS degree in mechanical engineering from the University of Nebraska-Lincoln in 2012 and an MS degree in aerospace engineering from the University of Michigan in 2015.

Dylan McKeithen is an optical engineer at the JPL, where he is a member of the High Contrast Imaging Group in the Optics Section. He received his BS degree in physics from the University of Southern California in 2019.

Stefan R. Martin is a senior optical engineer in the High Contrast Imaging Group in the Optics Section of the JPL. He received his BSc degree in physics from the University of Bristol and $\mathrm{PhD}$ in engineering from the University of Wales and has been with JPL since 1999.

John Steeves is an optical engineer in the Advanced Deployable Structures Group at the JPL. $\mathrm{He}$ received his $\mathrm{PhD}$ in aerospace engineering from Caltech in 2015 and has been at JPL ever since.

David Webb is the mechanical lead for starshade technology development at the JPL, where he is a member of the Advanced Deployable Structures Group. He received his BS degree in mechanical engineering from California Polytechnic State University, San Luis Obispo in California in 2007 and has been at JPL since 2007.

Douglas Lisman is the systems engineering lead for starshade technology development at the JPL, where he is a member of the Instrument Systems Engineering Group. He received his BS degree in mechanical engineering from Washington University in St. Louis in 1984 and has been at JPL since 1984 . 